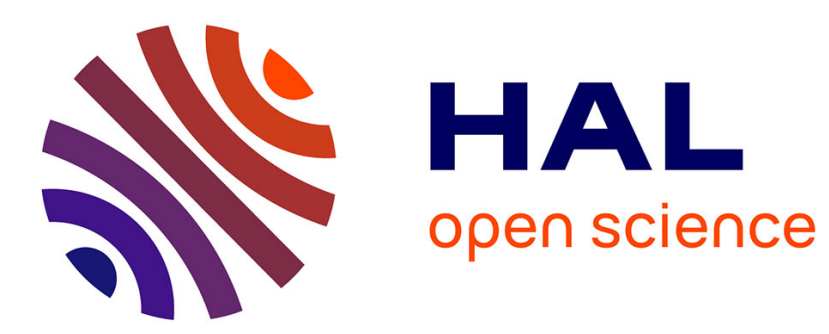

\title{
L'acquisition de la lecture en français: Etude longitudinale de la première à la seconde année du primaire
}

Liliane Sprenger-Charolles

\section{To cite this version:}

Liliane Sprenger-Charolles. L'acquisition de la lecture en français: Etude longitudinale de la première à la seconde année du primaire. Annee Psychologique, 1994, 94, pp.553-574. hal-00733594v2

\section{HAL Id: hal-00733594 \\ https://hal.science/hal-00733594v2}

Submitted on 24 Sep 2012

HAL is a multi-disciplinary open access archive for the deposit and dissemination of scientific research documents, whether they are published or not. The documents may come from teaching and research institutions in France or abroad, or from public or private research centers.
L'archive ouverte pluridisciplinaire HAL, est destinée au dépôt et à la diffusion de documents scientifiques de niveau recherche, publiés ou non, émanant des établissements d'enseignement et de recherche français ou étrangers, des laboratoires publics ou privés. 


\title{
Persée
}

\author{
http://www.persee.fr
}

\section{L'acquisition de la lecture en français : étude longitudinale de la première à la seconde année du primaire}

\section{Sprenger-Charolles}

L'Année psychologique, Année 1994, Volume 94, Numéro 4

p. $553-574$

\section{Voir l'article en ligne}

Résumé L'objectif de cette étude est d'examiner l'évolution des procédures de lecture chez des enfants francophones scolarisés en première et seconde année du primaire. Les sujets $(N=31)$ ont passé à trois reprises une épreuve de lecture de mots contrastés en fonction de la fréquence (mots fréquents vs mots rares) et de la régularité (mots réguliers simples, mots réguliers complexes contenant des digraphes et mots irréguliers). L'hypothèse centrale est que les enfants utilisent d'abord des procédures de lecture par médiation phonologique auxquelles vont se substituer progressivement, mais pas totalement, des procédures orthographiques. On escomptait donc, dans un premier temps, un effet de la régularité sans incidence de la fréquence, cette dernière ne devant interférer avec la régularité que dans les étapes ultérieures de l'acquisition. On attendait également une évolution en profondeur de la nature des unités de traitement utilisées : des unités de surface que sont les lettres aux unités plus profondes que sont les graphèmes. Les résultats ont, de fait, permis de voir que les procédures de lecture par médiation phonolcmque occupent une place importante, les meilleures performances se rencontrait toujours sur les mots réguliers. Ils ont également permis de relever une tendance développementale intéressante pour l'effet de la complexité : contrairement aux hypothèses, cet effet, qui s'atténue entre les sessions, est toujours au profit des mots complexes. Enfin, ils ont permis de constater un effet inattendu de la fréquence qui se manifeste de façon constante mais sans interagir avec la régularité. Ces résultats sont analysés en relation avec ceux obtenus dans un précédent volet de cette étude longitudinale. Mots-clés : acquisition de la lecture, médiation phonologique, procédures orthographiques, traitement des digraphes.

\section{Avertissement}

L'éditeur du site " PERSEE » - le Ministère de la jeunesse, de l'éducation nationale et de la recherche, Direction de l'enseignement supérieur, Sous-direction des bibliothèques et de la documentation - détient la propriété intellectuelle et les droits d'exploitation. A ce titre il est titulaire des droits d'auteur et du droit sui generis du producteur de bases de données sur ce site conformément à la loi n`98-536 du 1er juillet 1998 relative aux bases de données.

Les oeuvres reproduites sur le site « PERSEE » sont protégées par les dispositions générales du Code de la propriété intellectuelle.

Droits et devoirs des utilisateurs

Pour un usage strictement privé, la simple reproduction du contenu de ce site est libre.

Pour un usage scientifique ou pédagogique, à des fins de recherches, d'enseignement ou de communication excluant toute exploitation commerciale, la reproduction et la communication au public du contenu de ce site sont autorisées, sous réserve que celles-ci servent d'illustration, ne soient pas substantielles et ne soient pas expressément limitées (plans ou photographies). La mention Le Ministère de la jeunesse, de l'éducation nationale et de la recherche, Direction de l'enseignement supérieur, Sous-direction des bibliothèques et de la documentation sur chaque reproduction tirée du site est obligatoire ainsi que le nom de la revue et- lorsqu'ils sont indiqués - le nom de l'auteur et la référence du document reproduit. 
Toute autre reproduction ou communication au public, intégrale ou substantielle du contenu de ce site, par quelque procédé que ce soit, de l'éditeur original de l'oeuvre, de l'auteur et de ses ayants droit.

La reproduction et l'exploitation des photographies et des plans, y compris à des fins commerciales, doivent être autorisés par l'éditeur du site, Le Ministère de la jeunesse, de l'éducation nationale et de la recherche, Direction de l'enseignement supérieur, Sous-direction des bibliothèques et de la documentation (voir http://www.sup.adc.education.fr/bib/ ). La source et les crédits devront toujours être mentionnés. 
UFR de Linguistique

CNRS, URA 1031

Université Paris $V^{1}$

\section{L'ACQUISITION DE LA LECTURE EN FRANÇAIS : ÉTUDE LONGITUDINALE DE LA PREMIÈre À LA SECONDE ANNÉE du PRIMAIRE par Liliane SPRENGER-ChAROLlES ${ }^{2}$}

SUMMARY : Reading acquisition in French first and second graders : longitudinal evidence.

This study investigated reading acquisition in French-speaking school children. Subjects $(N=31)$ were tested in three sessions with a task that involved reading words that varied on frequency (high vs. low) and regularity (simple regular words, complex regular words containing digraphs, and irregular words). The central hypothesis was that children would first use reading procedures involving phonological mediation, and gradually, but not completely, would replace these with orthographic procedures. Thus, we expected at the first session an effect of regularity independant of frequency ; frequency was expected to interfere with regularity in the later stages of acquisition. Furthermore, under the assumption that alphabetical processing is primarily sequential, i.e. letter by letter, a complexity effect was predicted; in other words, subjects were expected to read simple regular words more accurately than complex regular words containing digraphs.

Overall, the role of phonological mediation was confirmed; the best performance occured in sessions with regular words. Contrary to our prediction, the complexity effect, which diminished between sessions, was

1. Laboratoire d'Études sur l'acquisition et la pathologie du langage chez l'enfant, LEAPLE, 12 rue Cujas, 75005 Paris.

2. Je remercie tout particulièrement $F$. Bresson pour une première lecture critique de cet article. 
always to the advantage of complex words. Finally, we noted an unexpected effect of frequency, which was apparent in the three sessions and did not interact with regularity. These results are examined with regard to results obtained in a earlier part of this longitudinal study.

Key words : reading acquisition, phonological mediation, orthographical procedures, digraph processing.

\section{INTRODUCTION}

Dans la reconnaissance des mots écrits, on distingue le plus souvent deux types de traitement : une procédure d'assemblage (voie indirecte de lecture) et une procédure d'adressage (voie directe de lecture). Dans le premier cas, la chaîne graphique est découpée en constituants graphémiques, chaque constituant étant mis en correspondance avec un phonème. Les phonèmes sont assemblés et le mot est prononcé sur la base de ce code phonologique prélexical. Dans le second cas, le mot écrit, apparié directement avec l'entrée lexicale correspondante, est prononcé sur la base d'une phonologie postlexicale. La plupart des modèles de lecture chez l'adulte s'accordent sur l'existence de ces deux procédures (modèles dual-route : cf. Humphrey et Evett, 1985, pour une revue ; voir aussi Paap et Noël, 1991, ainsi que Coltheart, Curtis et Atkins 1993), la voie directe étant empruntée le plus souvent, et spécialement pour les mots fréquents, tandis que la voie indirecte est utilisée pour la lecture de mots rares.

Dans l'acquisition de la lecture, l'utilisation de la voie indirecte et de la voie directe serait précédée par la mise en œuvre de procédures logographiques. De plus, d'après la plupart des modèles développementaux (cf. Frith, 1985, 1986, 1990 ; Morton, 1989 ; Harris et Coltheart, 1986 ; Marsh, Friedman, Welch et Desberg, 1981 ; Marsh, Friedman, Desberg et Saterdahl, 1981) ces trois procédures de traitement de l'information constitueraient des étapes successives de cette acquisition.

Ainsi, selon Frith, dans l'étape logographique les mots sont « identifiés » à partir d'indices contextuels - non linguistiques et d'indices visuels relatifs à leur forme globale ou à des configurations locales particulièrement saillantes. Sur cette base, se constitue un vocabulaire "visuel » dont l'étendue reste extrêmement limitée. La seconde étape, qui correspond à la voie 
indirecte, est dite alphabétique. L'enfant, en s'appuyant sur sa connaissance des lettres de l'alphabet, utilise les relations graphie-phonie pour lire. Cette lecture par médiation phonologique, qui est sensible à la régularité orthographique des items mais également à leur longueur en raison de son caractère strictement séquentiel, permet de lire des mots nouveaux qui n'ont jamais été rencontrés et, par là, de renforcer les connaissances des relations entre orthographe et phonologie. Elle conduit cependant à produire des erreurs de régularisation pour les mots irréguliers. Aussi, dans une dernière étape développementale, des modalités de traitement orthographique (cf. la voie directe de lecture) vont se substituer progressivement, mais pas totalement, aux modalités alphabétiques: les mots fréquents, réguliers et irréguliers, sont reconnus directement, sur leur seule base orthographique, alors que la médiation phonologique intervient pour les mots rares.

Ce modèle développemental en étapes a conduit à deux interprétations différentes pour l'aboutissement à un fonctionnement orthographique. Selon Morton (1989), seul le traitement alphabétique contribue à l'établissement de la voie directe ou lexicale. Dans cette perspective, dès lors qu'apparaît la procédure alphabétique, le traitement logographique n'a plus cours : la seconde procédure remplaçant entièrement la première. Par conséquent, durant la période alphabétique, on n'observe pas d'effet de fréquence : la régularité orthographique détermine entièrement les performances. A l'inverse, pour Seymour $(1986,1993)$ la voie directe s'établit à partir des procédures logographique et alphabétique : on observe alors un effet de fréquence et de régularité orthographique, ces deux facteurs interagissant dans les débuts de l'apprentissage.

L'hypothèse de Seymour est corroborée par les résultats qu'il a obtenus à partir d'une étude longitudinale (cf. en particulier, Seymour, 1993 ; Seymour et Bunce, 1993 ; Seymour et Evans, 1993). De nombreuses autres études ont cependant conduit à relativiser la contribution de l'étape logographique dans la construction du lexique orthographique (Masonheimer, Drum et Ehri, 1984 ; Ehri et Wilce, 1985 ; Stuart et Coltheart, 1988 ; Wimmer et Hummer, 1990). Ainsi, pour Stuart et Coltheart, les enfants qui ont un niveau de conscience phonologique élevé ont, dès le début de l'apprentissage de la lecture, essentiellement recours à la médiation phonologique pour lire. L'étude de Wimmer et Hummer (1990 ; voir aussi Wimmer, 1993) mon- 
tre en outre qu'il pourrait y avoir une incidence de la langue cible sur le développement des performances : dans une langue à orthographe très opaque comme l'anglais, le stade logographique pourrait être nécessaire à l'établissement de la procédure lexicale, et par conséquent perdurerait en même temps que la procédure alphabétique, ce qui ne semble pas être le cas de l'allemand.

Il est donc nécessaire d'examiner l'acquisition de la lecture en français, langue qui, comme l'anglais, a une orthographe souvent considérée comme opaque. En fait, les descriptions du système graphique du français montrent que les relations graphiephonie sont le plus souvent régulières à condition de prendre comme unité de base du système graphique le graphème, et non la lettre, en tenant compte de quelques règles contextuelles (cf. Gak, 1976 ; Catach, 1980) : ainsi, « ou », « au », « ch » ou « an » sont des graphèmes au même titre que « $a$ », « $s$ » ou « $c$ », ces deux derniers se prononçant respectivement / s / ou / z / et / s / ou / k / selon le contexte graphique.

Si le français a une écriture relativement régulière, on peut faire l'hypothèse que les enfants qui commencent à apprendre à lire s'appuieront essentiellement sur les relations graphie-phonie (cf. également Content, 1991, pour des résultats sur des lecteurs adultes francophones) : on devrait donc observer, dans les premiers temps de l'apprentissage de la lecture, un effet de la régularité orthographique mais pas de traces de traitements logographiques qui pourraient se manifester, entre autres, par la présence d'un effet de la fréquence.

De plus, si les enfants utilisent les relations graphie-phonie pour lire, on peut supposer que, au début de l'apprentissage de la lecture, ils mettront tout d'abord en ceuvre des traitements strictement séquentiels, lettre à lettre. Dans la plupart des modèles développementaux, et en particulier dans celui de Frith, cette question n'est pas abordée frontalement : il n'est en effet pas précisé si les premières unités de traitement sont les lettres en relation avec les sons ou les graphèmes en relation avec les phonèmes. Cependant la terminologie utilisée par Frith pour désigner l'étape de lecture par médiation phonologique - à savoir phase " alphabétique " - permet de penser que, pour elle, les unités de traitement appréhendées par les enfants sont plutôt les lettres. Si tel est bien le cas, on peut faire l'hypothèse que les enfants auront des difficultés y compris avec les mots réguliers dans la mesure où le système d'écriture 
du français comporte de nombreux graphèmes complexes ${ }^{3}$. Plus précisément, les enfants, dans un premier temps, devraient avoir des performances meilleures pour les mots réguliers ne contenant que des graphèmes simples que pour ceux contenant des graphèmes complexes.

De fait, les résultats de deux expériences portant sur des enfants francophones ${ }^{4}$ examinés au milieu et à la fin de la première année du primaire corroborent partiellement ces hypothèses (cf. Sprenger-Charolles et Casalis, à paraître). Ainsi, on a pu observer, dans ces deux expériences, un fort effet des régularités qui s'est manifesté par la supériorité des mots réguliers sur les irréguliers et par l'importance croissante des erreurs de régularisation. Ces résultats témoignent du fait que la médiation phonologique occupe une place importante dans les débuts de l'apprentissage de la lecture et que son rôle s'accentue entre le milieu et la fin de la première année du primaire.

On a observé quelques différences entre les deux expériences pour le traitement des graphèmes complexes. Les résultats de la première expérience rejoignaient ceux de Content et Leybaert (1992) : I'effet de la complexité, au profit des mots simples, s'atténuait entre les sessions. Ceux de la seconde expérience montraient une inversion de tendance : les mots composés uniquement de graphèmes simples étaient mieux lus que ceux comportant des graphèmes complexes lors de la première session, l'inverse étant observé pour la seconde session. Ces différences peuvent s'expliquer par le fait que les mots réguliers complexes utilisés dans la première expérience contenaient des digraphes et des trigraphes alors que, pour la seconde étude, n'ont été retenus que des items contenant des digraphes ne possédant, en outre, pas d'allographes plus simples (par exemple, « ou » et non « au »).

3. Outre les lettres géminées ( $1 \mathrm{ll} »)$, les graphèmes complexes les plus fréquents du français sont pour les voyelles : « ai », « au », « eu », « ou », « oi ", plus les nasales « an "/ « en", « in" / « en", « on», « un» et, pour les consonnes : « ch », " gn » et « qu ».

4. Une de ces deux expériences constitue le premier volet de la longitudinale présentée ci-après : la méthodologie était donc exactement identique à celle utilisée par la suite, la seule différence concernait l'effectif de la population ( 38 enfants : les 31 sujets de la présente étude plus 7 autres enfants qui ont disparu principalement en raison de déménagement). Pour l'autre expérience, qui portait sur 34 enfants de la même population, une méthodologie similaire a été utilisée, la seule différence concernait le matériel. 
Nous avons également pu relever, toujours dans les deux expériences, un effet inattendu de la fréquence qui était plus fort lors de la première session et, dans cette session, plus marqué pour les mots réguliers alors que l'inverse se produisait en juin. Le résultat obtenu pour la session de février n'est pas compatible avec l'hypothèse de traitements logographiques : en effet ces traitements ne sont, par définition, pas sensibles à la régularité des items. Une interprétation alternative a donc dû être trouvée. La possibilité d'obtenir une réponse correcte à partir d'un assemblage phonologique est la principale différence entre les mots réguliers et irréguliers. On peut donc penser que la fréquence interviendrait dans une seconde étape du traitement en interférant avec les mécanismes de lecture par mise en relation graphie-phonie : elle faciliterait l'accès aux mots réguliers qui pourraient être reconnus lors des premières étapes de l'apprentissage de la lecture, c'est-à-dire quand les mécanismes d'assemblage sont encore peu performants, via le lexique oral sur la base d'un assemblage partiel de leurs codes phonologiques d'entrée ce qui est possible pour les mots réguliers mais pas pour les irréguliers. Par contre, l'effet de la fréquence obtenu en juin était similaire à celui normalement relevé chez les adultes : il était plus fortement marqué sur les mots irréguliers ce qui a permis d'avancer l'hypothèse de la mise en place progressive d'un lexique orthographique. Il faut cependant noter que ce dernier résultat était entaché par des effets plafond pour les mots réguliers fréquents comme rares.

L'étude qui est l'objet du présent article se situe dans le prolongement d'une des deux expériences que nous venons de présenter. Elle a pour objectif de réexaminer la question de l'effet de la fréquence, de la régularité et de la complexité au cours des deux premières années de l'apprentissage de la lecture. La réalisation de cet objectif s'appuie sur l'analyse des réponses correctes et de leur temps de latence pour la lecture de mots dont on a fait varier la fréquence, la régularité et la complexité.

Pour atteindre notre objectif il nous a paru indispensable d'employer la méthode longitudinale (ce qui n'est pas le cas dans un certain nombre d'études portant sur l'acquisition de la lecture : cf. en particulier les travaux de Backman, Bruck, Hebert et Seidenberg, 1984 ; Waters, Seidenberg et Bruck, 1984) : en effet, lorsque les mêmes sujets sont testés à des pé- 
riodes différentes, les différences observées dans les facteurs sous-jacents aux performances renvoient bien à une dynamique développementale et ne peuvent être imputées à des différences de population.

Ce type d'étude longitudinale, portant sur les débuts de l'apprentissage de la lecture, pose cependant des problèmes méthodologiques importants. En effet, dans les toutes premières étapes de l'apprentissage de la lecture, il est possible de s'appuyer à la fois sur l'analyse des réponses correctes et sur celle des erreurs pour examiner la nature des procédures de traitement de l'information mises en cuvre par les sujets. Cependant, étant donné le faible nombre de réponses correctes obtenu alors, on ne peut utiliser l'analyse des temps de latence des réponses. Par contre, quand les enfants ont appris à lire, ce qui arrive très rapidement en général, le nombre de réponses correctes devient suffisamment important pour permettre d'analyser leur temps de latence. On ne peut donc utiliser exactement le même type d'indicateurs des procédures de traitement de l'information au début et à la fin d'une étude longitudinale portant sur l'apprentissage de la lecture dans les premières années du primaire.

Ainsi, dans notre étude longitudinale, les enfants ont été vus en tout quatre fois : au milieu et à la fin de la première et de la seconde année du primaire. Les analyses des résultats pour les deux premières sessions ont porté sur les réponses correctes et les erreurs (cf. Sprenger-Charolles et Casalis, à paraître), l'examen des temps de latence des réponses ne pouvant être utilisé pour la première session vu le faible nombre de réponses correctes alors que tel n'était pas le cas pour les trois sessions suivantes sur lesquelles porte le présent article.

\section{MÉTHODE}

\section{Matériel}

Les mots utilisés dans l'étude ont été sélectionnés en fonction de deux critères principaux : la régularité et la fréquence.

Trois niveaux de régularité ont été sélectionnés : mots réguliers simples, mots réguliers complexes et mots irréguliers. Un mot est dit régulier s'il ne comporte que des graphèmes fréquents et irrégulier s'il comporte un graphème rare (" porte " / port / versus « album » / albom /). 
La fréquence des graphèmes a été établie en référence aux travaux de Gak (1976) et de Catach (1980). Un mot régulier est dit simple si à chaque lettre, sauf le «e » muet, correspond un phonème (par exemple, «table »/tabl /). Les mots dits complexes incluent un bigraphe qui ne peut pas avoir de représentation graphique simple (par exemple, « ou » dans « route » / rut / ou « in » dans « matin » / matî /). De plus, les items réguliers, simples et complexes, ne comportent pas de graphèmes contextuels, c'est-à-dire des graphèmes dont la prononciation est fonction de l'environnement ( $\mathrm{c} », \ll \mathrm{g}$ »).

À chaque niveau de régularité, les mots ont été séparés en deux sous-classes en fonction de leur fréquence lexicale : mots fréquents et rares (« table » vs « sable "; « pied » vs « poêle »). Les mots fréquents ont été choisis parmi les 500 premiers items des « Listes Orthographiques de Base » (« LOB », Catach, 1984). Ces listes sont le résultat du croisement de plusieurs tables comportant des fréquences orales et écrites $^{5}$. Les mots rares ne sont pas dans les «LOB » mais sont présents dans les tables additives du «Français fondamental » (Gougenheim, Michea, Rivenc et Sauvageot, SD). Le choix de mots relativement peu rares s'explique par le fait que les items sélectionnés doivent être connus par les enfants les plus jeunes de l'étude (c'est-à-dire qu'ils n'aient pas, pour eux, le statut de non-mots). La familiarité orale de ces items a également été contrôlée auprès d'une population d'enfants de même âge ${ }^{6}$.

Les mots ont par ailleurs entre 4 et 8 lettres. La longueur de chacune des sous-listes étant strictement identique en nombre de lettres. La liste comprend 8 mots de chaque catégorie de régularité : 4 fréquents et 4 rares (cf. la présentation de la liste en annexe).

\section{Procédure}

On demande à chaque enfant de lire à haute voix les mots qui apparaissent successivement au centre d'un écran d'ordinateur. Une police de caractères $(7 \times 5 \mathrm{~mm})$ comparable à celle utilisée dans les manuels de lecture a été élaborée spécialement pour cette étude. L'ordinateur intègre une carte vocale qui permet l'enregistrement complet des réponses et le calcul des temps de latences. Nous n'avons pas utilisé de clé vocale pour calculer les temps de latence des réponses en raison des contraintes qu'impose cette méthodologie. En particulier, les sujets doivent avoir pour consigne de ne donner le mot que lorsqu'ils

5. Juilland, Brodin et Davidovitch (1970), Gougenheim, Michea, Rivenc et Sauvageot (1964), Trésor de la langue française (1971).

6. Nous avons essayé de sélectionner les mots en fonction de leur fréquence dans les manuels de lecture. Cela n'a pas été possible en raison des différences entre les manuels utilisés dans les différentes classes. 
sont sûrs de leur réponse et, de plus, cette réponse doit atteindre un certain seuil pour être correctement enregistrée par la clé vocale (cf. pour une analyse critique de l'utilisation de la clé vocale : Pechmann, Reetz et Zerbst 1989). Il ne nous a pas semblé possible de respecter ces deux contraintes dans un travail portant sur les débuts de l'acquisition de la lecture. C'est pour cette raison que nous avons choisi de travailler avec une carte vocale qui enregistre complètement les réponses et permet, à partir de l'observation du signal de la parole, de détecter le début effectif de la réponse correcte.

Les items-test sont présentés un par un en ordre semi-aléatoire. Une séquence de trois essais, renouvelable en cas de non compréhension de la consigne, précède la session de test qui dure approximativement 10 minutes et au cours de laquelle on ne donne aucune indication aux enfants sur la nature de leurs réponses. Pendant la session de test les réponses sont enregistrées par l'ordinateur et notées par l'expérimentateur. Les performances sont ensuite réexaminées à partir des enregistrements et leurs temps de latence sont alors calculés.

\section{Sujets}

Soixante enfants parlant le français en tant que langue maternelle, ne présentant pas de handicaps langagiers ou sensori-moteurs ni de troubles psychologiques détectés par les instituteurs, psychologues ou médecins scolaires, ont été testés à la fin de la dernière année de maternelle. Quarante de ces 60 sujets ont été sélectionnés en fonction de leur niveau cognitif et de leur niveau de lecture. Ont été retenus les sujets non-lecteurs ayant un niveau cognitif moyen : scores entre le $25^{\circ}$ et le $75^{\text {e }}$ centile aux Matrices progressives de Raven pour le niveau cognitif et scores inférieurs à ceux obtenus après un mois de scolarisation primaire d'après les résultats à un test de lecture (BATEL.EM, Savigny, 1974).

En première année du primaire, ces enfants étaient scolarisés dans 5 écoles situées dans un quartier de Paris comportant une population de milieu socio-économique moyen. Les enfants étaient alors répartis dans 12 classes utilisant des méthodes d'apprentissage de la lecture représentatives de la situation française : dix méthodes mixtes avec des variations dans la progression de l'enseignement explicite des règles de correspondance graphème-phonème, une méthode visuelle-globale et une méthode phonique. Cette diversité, non seulement de méthodes mais également des enseignants (un par classe), a pour conséquence d'annuler, au moins partiellement, l'effet des variables pédagogiques. Ces enfants ont été testés quatre fois : en février et en juin de la première et de la seconde année du primaire. Ne sont reportés dans l'article que les résultats des trois dernières sessions. La non intégration des résultats de la première session s'explique par le fait que nous n'avons pas pu calculer, pour cette session, les temps de latence des réponses correctes en raison de leur faible nombre. 


\section{PRÉDICTIONS}

Notre hypothèse centrale est que, dans un premier temps, les enfants ne devraient mettre en cuvre que des procédures alphabétiques de traitement de l'information graphique, sensibles à la régularité des items, alors que, dans un second temps, ils devraient avoir recours à des procédures orthographiques plus sensibles à la fréquence. En conséquence, on s'attend à observer, pour la (les) première(s) session(s), un nombre plus important de réponses correctes et des temps de latence des réponses plus courts pour les mots réguliers que pour les irréguliers, sans différence entre mots fréquents et rares, alors que, pour la (les) dernière(s) session(s), l'effet de la fréquence devrait interagir avec celui de la régularité, ce dernier facteur ne devant avoir une incidence que sur les mots rares : on devrait alors observer un nombre plus élevé de réponses correctes et des temps de latence des réponses plus courts pour les mots réguliers rares que pour les irréguliers rares ${ }^{7}$. En outre, si les traitements alphabétiques sont tout d'abord séquentiels, c'est-à-dire lettre à lettre, on devrait observer un effet de la complexité pour les mots réguliers qui devrait se modifier fortement entre les sessions : ainsi, pour la première session, les mots ne contenant que des graphèmes simples devraient être mieux lus et plus rapidement que ceux qui comportent des graphèmes complexes, l'inverse devant s'observer pour les deux dernières sessions.

\section{RÉSULTATS}

L'analyse de la variance a porté sur les facteurs suivants : session (juin de la première année du primaire ainsi que février

7. On rend compte de cette différence, dans le cadre des modèles « dual route ", en évoquant le rôle de la voie directe par rapport à celui de la voie indirecte - par correspondance graphie-phonie - et le fait que les traitements par voie directe sont plus rapides que ceux par voie indirecte : les mots fréquents seraient accédés rapidement, uniquement par voie directe, qu'ils soient réguliers ou irréguliers, alors que l'accès aux mots rares s'effectuerait plus lentement ce qui permet une réponse par voie directe et par voie indirecte. Or pour les mots irréguliers les réponses obtenues par voie directe et par voie indirecte ne concordent pas. Ce conflit permet de rendre compte de l'allongement des temps de latence des réponses pour les mots irréguliers rares comparativement aux réguliers rares. 
et juin de la seconde année), régularité (réguliers simples, réguliers complexes et irréguliers), fréquence (fréquents et rares). Quand un effet principal de la régularité émerge, on a effectué les deux contrastes suivants : réguliers simples contre réguliers complexes (effet de la complexité) et réguliers simples et complexes contre irréguliers (effet de l'irrégularité). Dans la mesure où certains enfants ont été absents pour une session, les données ne prennent en compte que 31 sujets pour l'analyse des réponses correctes. Les analyses pour les temps de latence des réponses portent sur 24 à 31 sujets selon les sessions. Cette différence d'effectif s'explique par le fait que les données avec valeur manquante ne sont pas incluses dans les analyses de la variance. Lors de la première session les enfants avaient en moyenne 83,93 mois (écart-type : 2,75).

\section{RÉPONSES CORRECTES}

Les performances s'améliorent entre les sessions : $79 \%$ de réponses correctes pour la première session contre $90 \%$ et $92 \%$ pour les seconde et troisième sessions (cf. tableau I). Ce gain est reflété par une différence significative entre sessions $(F(2,60)=41.02, p=.0001)$. On observe un effet principal de la régularité $(F(2,60)=131.21, p=.0001)$ s'expliquant par le fait que, d'une part, les mots réguliers simples et complexes sont mieux lus que les irréguliers (effet de l'irrégularité : $F(1,30)=137.75$, $\mathrm{p}=.0001$ ) et que, d'autre part, les mots réguliers complexes sont mieux lus que les simples (effet de la complexité : $F(1,30)=6.85$,

\begin{tabular}{|l|l|l|l|l|}
\hline Session & fréquence & $\begin{array}{l}\text { Régulier } \\
\text { simple }\end{array}$ & $\begin{array}{l}\text { Régulier } \\
\text { complexe }\end{array}$ & Irrégulier \\
\hline S1 (1P06) & Fréquent & $3.93(0.25)$ & $3.97(0.18)$ & $1.97(1.25)$ \\
& Rare & $3.68(0.65)$ & $3.90(0.30)$ & $1.42(0.92)$ \\
\hline S2 (2P02) & Fréquent & $3.97(0.18)$ & $4.00(0.00)$ & $3.35(1.05)$ \\
& Rare & $3.87(0.34)$ & $3.93(0.25)$ & $2.55(0.89)$ \\
\hline S3 (2P06) & Fréquent & $3.84(0.37)$ & $4.00(0.00)$ & $3.74(0.51)$ \\
& Rare & $3.87(0.34)$ & $3.81(0.40)$ & $2.71(0.97)$ \\
\hline
\end{tabular}

TABl.EAU I. - Réponses correctes (moyenne sur un maximum de 4 et écart typej.

Correct responses (mean on maximum 4 and standard deviation). 
$\mathrm{p}=.013$ ). De plus, l'incidence de la régularité s'atténue entre les sessions (interaction session * régularité : $F(4,120)=49.39$, $\mathrm{p}=.0001$ ). Des analyses complémentaires indiquent que l'effet de l'irrégularité a une incidence à l'intérieur des trois sessions (respectivement pour les sessions un, deux et trois : $F(1,30)=$ $196.85, \mathrm{p}=.0001 ; \mathrm{F}(1,30)=45.05, \mathrm{p}=.0001 ; \mathrm{F}(1,30)=38.87$, $\mathbf{p}=.0001)$ alors que celui de la complexité n'est significatif que pour la première session $(F(1,30)=3.89 ; p=.058, F(1,30)$ $=1.85$, NS $; \mathrm{F}(1,30)=0.81$, NS). Ces différentes tendances sont cependant entachées par des effets plafond pour les mots réguliers simples et complexes (respectivement $95 \%$ et $98 \%$ de réponses correctes).

Contrairement aux prédictions, on observe un effet de la fréquence : les mots rares sont moins bien lus que les fréquents $(F(1,30)=70.69, p=.0001)$ sans changement entre les sessions $(F(2,60)=0.65$, NS). Cependant, la fréquence interagit avec la régularité $(F(2,60)=37.44, p=.0001)$, la triple interaction session * régularité * fréquence étant également significative $(F(4,120)=2.81, p=.028)$ : l'effet de la fréquence, qui est surtout marqué sur les mots irréguliers, s'accentue à ce niveau entre la première session et les deux suivantes. Cette tendance peut cependant s'expliquer par les effets plafond obtenus pour les mots réguliers fréquents et rares : respectivement $99 \%$ de réponses correctes pour les mots fréquents et $95 \%$ pour les rares.

\section{TEMPS DE LATENCE DES RÉPONSES CORRECTES}

Comme pour les réponses correctes, les performances s'améliorent entre les sessions : les temps sont en moyenne de plus de 2 secondes pour la première session contre un peu plus ou un peu moins d'une seconde respectivement pour la deuxième et la troisième session (cf. tableau II). Ce gain est reflété par une différence significative entre sessions $(F(2,46)=67.78, p=.0001)$. On observe un effet principal de la régularité $(\mathrm{F}(2,46)=23.36$, $\mathrm{p}=.0001$ ) s'expliquant, comme pour les réponses correctes, par un effet de l'irrégularité et de la complexité : les mots réguliers simples et complexes sont lus plus rapidement que les irréguliers $(\mathrm{F}(1,23)=25.45, \mathrm{p}=.0001)$ tandis que les mots réguliers complexes sont lus plus rapidement que les simples $(F(1,30)=7.97, p=.008)$. De plus, l'effet principal de la régularité varie en fonction de la session $(F(4,92)=6.65, p=.0001)$. 


\begin{tabular}{|l|l|l|l|l|}
\hline Session & Fréquence & $\begin{array}{l}\text { Régulier } \\
\text { simple }\end{array}$ & $\begin{array}{l}\text { Régulier } \\
\text { complexe }\end{array}$ & Irrégulier \\
\hline S1 (1P06) & Fréquent & $\begin{array}{l}1656(721) \\
2400(1130)\end{array}$ & $\begin{array}{l}1458(561) \\
2141(1006)\end{array}$ & $2636(1818)$ \\
& Rare & $8338(2169)$ \\
\hline S2 (2P02) & Fréquent & $\begin{array}{l}851(276) \\
\text { Rare }\end{array}$ & $\begin{array}{l}900(329) \\
1238(504)\end{array}$ & $1106(551)$ \\
& Fréquent & $750(241)$ & $758(281)$ & $897(451)$ \\
S3 (2P06) & Rare & $947(363)$ & $938(488)$ & $1139(373)$ \\
\hline
\end{tabular}

TABLEAU II. - Temps de latence des réponses correctes (moyenne en millisecondes et écart type).

Latency of correct responses (mean in milliseconds and standard deviation).

Des analyses complémentaires indiquent une différence significative entre mots réguliers simples / complexes versus irréguliers pour chacune des trois sessions (respectivement pour les sessions 1,2 et $3: F(1,24)=14.65, p=.0008 ; F(1,28)=24.37$, $p=.0001 ; F(1,29)=13.56, p=.0001)$. Par contre, la différence entre mots complexes et simples n'est significative qu'à l'intérieur des deux premières sessions (respectivement : $F(1,30)=$ $5.29, \mathrm{p}=.028 ; \mathrm{F}(1,30)=3.30, \mathrm{p}=.079 ; \mathrm{F}(1,30)=0.00, \mathrm{NS})$.

On observe également un effet de la fréquence : les mots rares sont lus plus lentement que les fréquents $(F(1,23)=56.77$, $p=.0001)$ et cet effet diminue entre les sessions $(F(2,46)=5.28$, $\mathrm{p}=.008$ ) sans changement global en fonction de la régularité $(\mathrm{F}(2,46)=0.23, \mathrm{NS})$. La triple interaction session * régularité * fréquence n'est pas significative $(F(4,92)=0.43$, NS).

\section{DISCUSSION}

L'analyse des réponses correctes et celle de leur temps de latence montrent un effet de la régularité s'expliquant, dans les deux cas, par une différence en faveur des mots réguliers qui sont de façon constante mieux lus et plus rapidement que les irréguliers. Ce résultat indique que les enfants, à cette étape de l'acquisition, s'appuient toujours fortement sur la médiation phonologique pour lire. 
Contrairement aux prédictions, les mots complexes sont mieux lus et plus rapidement que les simples, cet effet s'atténuant entre les sessions. Une explication possible de la supériorité des mots complexes est que, si les enfants traitent les bigraphes en tant qu'entité, ils ont moins d'unités à assembler dans le cas des mots complexes dans la mesure où ces items ont, par rapport aux mots simples de même longueur, un plus petit nombre de graphèmes : par exemple, quatre pour $\ll / t / a / b / 1$ $/$ (e) » contre trois pour «/r/ou / $/$ / (e) ». L'analyse des réponses correctes produites par le même groupe de sujets ${ }^{8}$, et avec la même liste de mots, lors d'une session antérieure (février de la première année du primaire : cf. la seconde expérience in Sprenger-Charolles et Casalis, à paraître et ci-dessus) avait par contre permis de voir que l'effet de la complexité était alors au profit des mots simples.

Ces deux résultats corroborent l'hypothèse que dans un premier temps, les enfants traiteraient les graphèmes complexes - ou tout au moins ceux qui sont utilisés dans la présente étude - comme des suites de lettres et, dans un second temps, comme des entités. Cette explication ne permet cependant pas de rendre compte de la baisse surprenante de l'effet de la complexité observée entre la fin de la première et de la seconde année du primaire. Une explication alternative doit donc être recherchée.

On peut penser qu'il y aurait, non pas deux mais trois sousprocessus impliqués dans la lecture par médiation phonologique : l'assemblage des codes phonologiques qui seraient ensuite transformés en codes articulatoires, les effets de ces deux composantes « cascadant » jusqu'aux traitements postlexicaux (cf. Marmurek et Rinaldo, 1992 ; Besner, 1987 ; Jared et Seidenberg, 1990) et évoluant en fonction du niveau de lecture. En particulier, la programmation des codes articulatoires jouerait un rôle important dans la lecture par médiation phonologique chez les sujets les plus jeunes ou les moins performants, son effet s'estompant avec l'amélioration du niveau de lecture (cf. Marmurek et Rinaldo, 1992 ; Besner, 1987). De même, on peut supposer qu'avec l'amélioration du niveau de lecture, les opérations d'assemblage deviendront moins coûteuses.

8. Les 31 sujets de la présente étude plus sept autres enfants qui ont disparu dans la suite de l'étude longitudinale. 
Les résultats obtenus pour les mots simples et complexes lors de la session de juin de la première année du primaire pourraient donc s'expliquer par le fait que, en raison du niveau de lecture encore faible des enfants, d'une part, les opérations d'assemblage doivent être plus coûteuses pour les mots simples dans la mesure où ils comportent un nombre plus élevé de graphèmes que les mots complexes et que, d'autre part, le temps de programmation de leurs codes articulatoires doit être plus long en raison de leur nombre plus élevé de phonèmes. On peut ajouter que les phonèmes des mots simples sont insérés dans des structures syllabiques complexes (cf. la présence de groupes consonantiques dans tous les items simples, à l'exception de " madame ", alors que les mots complexes ne comportent pas de suite de consonnes). Or les enfants ont des difficultés pour le traitement des groupes consonantiques (cf. Treiman, 1991). La baisse de l'effet de la complexité entre la fin de la première et de la seconde année du primaire serait alors due à une baisse du poids des opérations d'assemblage des codes phonologiques et de programmation des codes articulatoires consécutive à l'amélioration du niveau de lecture qui aurait pour conséquence une diminution de la double pénalisation des mots simples.

Si les effets de la régularité apparaissent de façon relativement convergente dans l'analyse des réponses correctes et dans celle des temps de latence des réponses, il n'en va pas de même pour l'effet de la fréquence : dans les deux cas, on a observé un effet principal de la fréquence mais tous les effets d'interactions divergent. Ainsi, pour les réponses correctes, on a constaté une interaction entre régularité et fréquence ainsi qu'entre régularité, fréquence et session. Ces interactions sont cependant biaisées, dès la session de juin de la première année du primaire, par des effets plafond pour les mots réguliers fréquents comme rares. Il est donc plus sage de s'appuyer sur la seule analyse des temps de latence des réponses pour interpréter l'effet de la fréquence. Or cette analyse indique simplement que l'effet principal de la fréquence diminue entre les sessions. Elle ne montre pas, contrairement à ce que l'on observe en général chez les adultes, une interaction entre fréquence et régularité s'expliquant par une absence d'effet de la régularité pour les mots fréquents mais pas pour les rares. La présence de cet effet de la fréquence, qui contredit l'hypothèse de traitements strictement alphabétiques (cf. Morton, 1989), 
n'est donc pas compatible avec celle de traitements identiques à ceux relevés chez les adultes.

L'analyse des réponses correctes produites lors de la session de février de la première année du primaire (cf. la seconde expérience in Sprenger-Charolles et Casalis, à paraître et ci-dessus) par les mêmes enfants ${ }^{9}$, et pour la même liste de mots, avait donné un résultat différent : l'effet de la fréquence était alors plus fortement marqué sur les mots réguliers. Ce résultat, qui ne permettait pas de corroborer l'hypothèse de traitements logographiques précoces dans la mesure où ces traitements ne doivent pas être sensibles à la régularité des items, était plus proche de ceux obtenus par Wimmer et Hummer (1990) que par Seymour (1993). On avait alors rendu compte de l'interaction observée entre fréquence et régularité par une argumentation voisine de celle proposée pour expliquer les effets de la complexité. Le processus de lecture par médiation phonologique impliquerait deux opérations, l'assemblage des codes phonologiques d'entrée suivi par la récupération des codes postlexicaux, la prononciation des mots s'effectuant sur cette dernière base et non sur celle de l'assemblage uniquement. Le résultat obtenu en février de la première année du primaire pourrait s'expliquer par le fait que les enfants auraient accès aux mots réguliers via le lexique oral à partir d'un assemblage partiel de leurs codes phonologiques d'entrée : par exemple, l'assemblage des trois premières lettres de «table " peut permettre la sélection de l'item correct. Cette opération, impossible pour les mots irréguliers, serait facilitée pour les mots fréquents réguliers dans la mesure où leurs codes phonologiques de sortie sont plus disponibles que ceux des mots réguliers rares.

A partir du mois de juin de la première année du primaire, l'interaction entre fréquence et régularité disparaît : l'effet principal de la fréquence diminuant simplement dans le temps. Ce résultat peut éventuellement s'expliquer par le fait que tous les mots seraient d'abord traités systématiquement par médiation phonologique puis " accédés » via le lexique oral.

Pour les items réguliers, le résultat de l'assemblage permet de trouver rapidement un candidat dans ce lexique, et ce plus rapidement pour les mots fréquents. La baisse de l'effet de la

9. Voir note 8 . 
fréquence entre la fin de la première et de la seconde année du primaire pour les mots réguliers pourrait alors être la manifestation du fait que, dans un premier temps, comme cela a été relevé pour la session de février de la première année du primaire, les mots fréquents réguliers seraient encore « accédés " via le lexique oral à partir d'un assemblage partiel de leurs codes phonologiques. Dans un second temps, les opérations d'assemblage s'automatisant, deviennent plus exhaustives : l'effet facilitateur de l'assemblage partiel pour les mots fréquents réguliers disparaîtrait alors et ne subsisterait que l'effet de la fréquence via le lexique oral.

Pour les mots irréguliers, il y aurait également une mise en cuvre des opérations d'assemblage qui conduisent à une erreur de régularisation aboutissant à la production d'un logatome. Un nouveau traitement serait alors engagé pour rechercher un candidat lexical. Ce retraitement se manifeste par un allongement des temps de latence des réponses pour les mots irréguliers fréquents et rares. La différence en fonction de la fréquence pourrait s'expliquer par le fait que le candidat lexical, qui serait recherché à partir de la réponse obtenue par assemblage (par exemple, de / môsjø / à / məsjø / ou de / fam / à / fam $/$, pourrait être plus facilement trouvé pour les mots irréguliers fréquents. On peut éventuellement rendre compte de la baisse de l'effet de la fréquence par le fait que, comme pour les mots réguliers, les opérations d'assemblage s'automatisent progressivement et deviennent plus exhaustives alors que dans un premier temps elles seraient encore partielles.

\section{CONCLUSION}

En résumé, on peut dire que les résultats de l'étude longitudinale reportés dans cet article et ceux du précédent volet de cette étude ont permis de voir que les procédures de lecture par médiation phonologique occupent une place importante au cours des deux premières années de l'apprentissage de la lecture, les meilleures performances se rencontrant toujours sur les mots réguliers.

Ils ont également permis de relever une tendance développementale intéressante pour l'effet de la complexité, qui s'inverse tout d'abord pour s'atténuer par la suite. Les enfants traiteraient donc, dans un tout premier temps, les graphèmes 
complexes comme des suites de lettres et, dans un second temps, comme des entités. On a rendu compte de la baisse de l'effet de la complexité entre les sessions de juin de la première et de la seconde année du primaire par la diminution probable du poids des opérations d'assemblage des codes phonologiques d'entrée et de programmation des codes articulatoires consécutive à l'amélioration de niveau de lecture : ces opérations pouvant pénaliser provisoirement les mots qui ne comportent pas de graphèmes complexes, à savoir les mots dits simples, dans la mesure où ils sont plus longs au niveau graphémique et phonémique que les mots complexes.

Enfin, dans les deux volets de cette étude on a constaté un effet inattendu de la fréquence. Cet effet se manifeste, dans les tout premiers temps, plus fortement sur les mots réguliers ce qui n'est pas compatible avec l'hypothèse de traitements précoces purement logographiques. Par la suite, l'interaction entre fréquence et régularité disparaît, l'effet principal de la fréquence diminuant simplement dans le temps. La présence de cet effet de la fréquence contredit cependant l'hypothèse de traitements strictement alphabétiques tandis que l'absence d'interaction entre fréquence et régularité ne permet pas de corroborer celle de traitements par voie directe. Ces résultats ne semblent pouvoir s'interpréter que si on postule que, dans cette étape de l'acquisition de la lecture, la mise en œuvre systématique de traitements par médiation phonologique serait suivie par celle de traitements postlexicaux : après assemblage - complet ou partiel - des codes phonologiques d'entrée, il y aurait récupération des codes postlexicaux, la prononciation des mots s'effectuant sur cette dernière base et non sur celle de l'assemblage uniquement.

ANNEXES : Liste des mots

Mots fréquents

\begin{tabular}{|l|l|l|}
\hline Réguliers simples & Réguliers complexes & Irréguliers \\
\hline porte & soir & pays \\
\hline table & route & pied \\
\hline livre & matin & femme \\
\hline madame & voiture & monsieur \\
\hline
\end{tabular}


Mots rares

\begin{tabular}{|l|l|l|}
\hline Réguliers simples & Réguliers complexes & Irréguliers \\
\hline barbe & voile & noyau \\
\hline sable & boule & poêle \\
\hline brune & patin & album \\
\hline brutal & tiroir & oignon \\
\hline
\end{tabular}

\section{$R \hat{E} S U M \hat{E}$}

L'objectif de cette étude est d'examiner l'évolution des procédures de lecture chez des enfants francophones scolarisés en première et seconde année du primaire. Les sujets $(N=31)$ ont passé à trois reprises une épreuve de lecture de mots contrastés en fonction de la fréquence (mots fréquents us mots rares) et de la régularité (mots réguliers simples, mots réguliers complexes contenant des digraphes et mots irréguliers). L'hypothèse centrale est que les enfants utilisent d'abord des procédures de lecture par médiation phonologique auxquelles vont se substituer progressivement, mais pas totalement, des procédures orthographiques. On escomptait donc, dans un premier temps, un effet de la régularité sans incidence de la fréquence, cette dernière ne devant interférer avec la régularité que dans les étapes ultérieures de l'acquisition. On attendait également une évolution en profondeur de la nature des unités de traitement utilisées : des unités de surface que sont les livitos aux unités plus profondes que sont les graphèmes. Les résultats ont, de fait, permis de voir que les procédures de lecture par médiation phonol vique occupent une place importante, les meilleures performances se rencioirant toujours sur les mots réguliers. Ils ont également permis de relever unt' tendance développementale intéressante pour l'effet de la complexité : non. trairement aux hypothèses, cet effet, qui s'atténue entre les sessions, est toujours au profit des mots complexes. Enfin, ils ont permis de constater un effet inattendu de la fréquence qui se manifeste de façon constante mais sans interagir avec la régularité. Ces résultats sont analysés en relation avec ceux obtenus dans un précédent volet de cette étude longitudinale.

Mots-clés: acquisition de la lecture, médiation phonologique, procédures orthographiques, traitement des digraphes. 


\section{BIBLIOGRAPHIE}

Backman J., Bruck M., Hebert M., Seidenberg M. S. - (1984) Acquisition and use of spelling sound correspondances in reading, Journal of Experimental Child Psychology, 38, 114-133.

Besner D. - (1987) Phonology, lexical access in reading and articulatory suppression : A critical review, The Quarterly Journal of Experimental Psychology, 39A, 467-478.

Catach N. - (1980). L'orthographe française : Traité théorique et pratique, Paris, Nathan (rééditions en 1986 et 1989).

Catach N. - (1984) Les listes orthographiques de base du français (LOB) : les mots les plus fréquents et leurs formes fléchies les plus fréquentes, Paris, Nathan.

Coltheart M., Curtis B., Atkins P., Haller M. - (1993). Dual route and parallel processing approaches, Psychological Review, 100, 589-608.

Content A. - (1991) The effect of spelling-to-sound regularity on naming in French, Psychological Research, 53, 3-12.

Content A., Leybaert J. - (1992) L'acquisition de la lecture : influence des méthodes d'acquisition, In P. Lecocq (Edit.) La lecture, processus, apprentissage, troubles, Lille, Presses Universitaires de Lille, 181-211.

Ehri L., Wilce L. S. - (1985) Movement into reading : Is the first stage of printed word learning visual or phonetic ? Reading Research Quarterly, 20, 163-179.

Frith U. - (1985) Beneath the surface of developmental dyslexia, In K. E. Patterson, J. C. Marshall et M. Coltheart (Edit.), Surface dyslexia : Cognitive and neuropsychological studies of phonological reading, Londres, Erlbaum, 301-330.

Frith U. - (1986) A Developmental framework for developmental dyslexia, Annals of Dyslexia, 36, 69-81.

Frith U. - (1990) Dyslexia as a developmental disorder of language, Londres, MRC, Cognitive Development Unit (non publié).

Gak V. G. - (1976) L'orthographe du français, essai de description théorique et pratique, Paris, Selaf.

Gougenheim G., Michea R., Rivenc P., Sauvageot A. - (1964) L'élaboration du français fondamental (1er degré): étude sur l'établissement d'un vocabulaire et d'une grammaire de base, Paris, Didier.

Gougenheim G., Michea R., Rivenc P., Sauvageot A. - (s.d.) Additif aux listes de fréquence : mots de fréquence $1 \grave{a} 19$, Tapuscrit, non publié.

Harris M., Coltheart M. - (1986) Language processing in children and adults : An introduction, Londres, Routledge \& Kegan Paul.

Humphreys G. W., Evett L. J. - (1985) Are there independent lexical and nonlexical routes in word processing? An evaluation of the dual-route theory of reading, The Behavioral and Brain Sciences, 8, 689-740.

Jared D., Seidenberg M. S. - (1990) Naming multisyllabic words, Journal of Experimental Psychology : Human Perception and Performance, 16, 92-105.

Juilland A., Brodin D., Davidovitch C. - (1970) Frequency dictionary of French words, La Haye, Mouton.

Marmurek H. H. C., Rinaldo R. - (1992) The development of letter and syllable effects in categorisation, reading aloud and picture naming, Journal of Experimental Child Psychology, 53, 277-299. 
Marsh G., Friedman M. P., Desberg P., Saterdahl K. - (1981) Comparison of reading and spelling strategies in normal and reading desabled children, In M. P. Friedman, J. P. Dass et N. O'Connors (Edit.), Intelligence and learning, New York, Plenum Press, 363-367.

Marsh G., Friedman M., Welch V., Desberg P. - (1981) A Cognitive developemental theory of reading acquisition, $I n$ G. E. Mackinnon et T. G. Waller (Edit.), Reading research: Advances in theory and practice, vol. 3, New York, Londres, Academic Press, 199-221.

Masonheimer P. E., Drum P. A., Ehri L. C. - (1984) Does environmental print identification lead children into word reading? Journal of Reading Behavior, 16, 257-271.

Morton J. - (1989) An Information-processing account of reading acquisition, In A. M. Galaburda (Edit.), From reading to neurons, Cambridge (MA), Bradford Book, MIT Press, 43-66.

Paap K. R., Noel R. W. - (1991) Dual-route models of print to sound : Still a good horse race, Psychological Research, 53, 13-24.

Savigny M. - (1974) Manuel (forme B) pour l'utilisation des tests BATELEM, Distribué par les FAP (Editions scientifiques et psychologiques), Issy les Moulineaux.

Pechmann T., Reetz T., Zerbst D. - (1989) Kritik einer Messmethode : Zur Ungenauigkeit von voice-key messungen, Sprache und kognition, 8, 65-71.

Seymour P. H. K. - (1986) Cognitive analysis of dyslexia, Londres, Routledge \& Kegan Paul.

Seymour P. H. K. - (1993) Un modèle du développement orthographique à double fondation, In J. P. Jaffré, L. Sprenger-Charolles et M. Fayol (Edit.), Apprentissage de la lecture et de l'écriture; Actes du colloque du Ministère de l'Education et de la Culture, Paris, Nathan, 57-79.

Seymour P. H. K., Bunce F. - (1993) Application of cognitive model to remediation in cases of developmental dyslexia, In J. Riddoch et $\mathrm{G}$. Humphreys (Edit.), Cognitive neuropsychology and cognitive rehabilitation, Hillsdale, Erlbaum.

Seymour P. H. K., Evans H. M. - (1993) The visual (orthographic) processor and developmental dyslexia, In D. Willows, R. S. Krug et E. Corcos (Edit.), Visual processes in reading and reading disabilities, Hillsdale (NJ), Erlbaum, 347-376.

Sprenger-Charolles L., Casalis S. - (à paraître) Reading and spelling acquisition in French first graders : Longitudinal evidence. Reading and writing.

Stuart M., Coltheart M. - (1988) Does reading develop in a sequence of stages? Cognition, 30, 139-151.

Treiman R. - (1991) Children's spelling errors on syllable-initial consonant cluster, Journal of Educational Psychology. 83, 3, 346-360.

Trésor de la langue française - (1971) Nancy, Centre National de la Recherche Scientifique.

Waters G. S., Seidenberg M. S., Bruck M. - (1984) Children's and adults' use of spelling sound information in three reading tasks, Memory and Cognition, 12, 293-305.

Wimmer H. - (1993) L'acquisition de la lecture dans une orthographe plus régulière que l'anglais : points de divergence, In J. P. Jaffré, L. SprengerCharolles et M. Fayol (Edit.), Apprentissage de la lecture et de l'écriture; Actes du colloque du Ministère de l'Education et de la Culture, Paris, Nathan, 97-106. 
Wimmer H., Hummer P. - (1990) How German speaking first graders read and spell : Doubts on the importance of the logographic stage, Applied Psycholinguistics, 11, 349-368.

(Accepté le 25 mai 1994.) 